\title{
Intermittency in Plasma Turbulence
}

\author{
V. Carbone, P. Giuliani, L. Sorriso-Valvo, P. Veltri \\ Dipartimento di Fisica, Universitá della Calabria, 87036 Roges di Rende, Italy and \\ Istituto Nazionale per la Fisica della Materia, Unitá di Cosenza, Italy \\ R. Bruno \\ Istituto di Fisica dello Spazio Interplanetario/CNR c.p. 27, 00044 Frascati, Italy \\ E. Martines ${ }^{1}$, V. Antoni ${ }^{1,2}$ \\ ${ }^{1}$ Consorzio RFX, Corso Stati Uniti, Padova, Italy \\ ${ }^{2}$ Istituto Nazionale per la Fisica della Materia, Unitá di Padova, Italy
}

\begin{abstract}
Intermittency in fluid turbulence can be evidentiated through the analysis of Probability Distribution Functions (PDF) for velocity fluctuations, which display a strong non-gaussian behavior at small scales. In this paper we investigate the occurrence of intermittency in plasma turbulence by studying the departure from the gaussian distribution of PDF for both velocity and magnetic fluctuations. We use data coming from two different experiments, namely in situ satellite observations of the inner solar wind and turbulent fluctuations in a magnetically confined fusion plasma. Moreover we investigate also time intermittency observed in a simplified shell model which mimics 3D MHD equations. We found that the departure from a gaussian distribution is the main characteristic of all cases. The scaling behaviour of PDFs are then investigated by using two different models built up in the past years, in order to capture the essence of intermittency in turbulence.
\end{abstract}

\section{Introduction}

The statistics of turbulent fluid flows can be characterised by the Probability Distribution Function(PDF) of velocity differences $\delta u_{r}=u(x+r)-u(x)$ over varying scales $r$ (see 1 and references therein). At large scales the PDF is approximately gaussian, as the scale decreases, the wings of the distribution become increasingly stretched, so that large deviations from the average value are present. This phenomenon is usually ascribed to intermittency. The way the PDF departs from the gaussian is very interesting because this has to do with models for intermittency in turbulence. If we introduce a scaling for fluctuations, namely $\delta u_{r} \sim r^{h}$ as the fluid equations indicate, a change of scale $r \rightarrow \operatorname{lr}$ (with $l>0$ ) leads to

$$
\delta u_{l r}=l^{h} \delta u_{r}
$$

This is interpreted as an equality in law 1 , that is the right-hand-side has the same statistical properties as the left-hand-side. It can be easily shown that if 
$h$ is unique, that is in a pure self-similar (fractal) situation, the standardized variables $\delta u_{r} /\left\langle\delta u_{r}^{2}\right\rangle^{1 / 2}$ have the same PDF for all scales $\tau$. On the contrary me must invoke the multifractal theory (or some other models) to describe intermittency, by conjecturing the presence of an entire range of values of $h$.

Intermittency has been observed some time ago and deeply investigated in fluid flows 1 , and more recently also in Magnetohydrodynamic (MHD) flows (see for example 2). In particular, experimental studies carried out in MHD flows deal mainly by analyses of satellite measurements of solar wing Huctuations

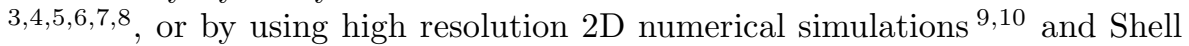
Models 11,122. All these analysis deal with the scaling exponents of structure functions, aimed to show that they follow anomalous scaling laws which can be compared with the usual energy cascade models for turbulence.

The non gaussian nature of PDF in MHD solar wind turbulence has been evidenced by Marsch_and $\mathrm{Tu} 13$, followed by quantitative analysis made by Sorriso-Valvo et al. 14. In fact, in order to investigate the properties of intermittency through the analysis of non gaussian character of PDF, it would be necessary to quantify the departure of PDF from gaussian statistics and to analyse how this departure depends on the scale $\tau$. In this work we present two models, introduced earlier to describe intermittency in fluid flows. Both models describe the evolution of PDF with $\tau$, by allowing some free parameters to evolve with the scale $\tau$. We then analyse some data of plasma turbulence coming from very different situations. First of all we show that both models are able to capture time intermittency observed in a discrete model which describes the MHD turbulent cascade (Section 3), then we show that also intermittency in real MHD turbulence, say the turbulence observed in the Solar Wind (Section 4) and magnetic turbulence as observed in a fusion device (Section 5), is described by the models. We will describe intermittency in plasma turbulence through the scaling laws of the characteristic parameters of both models. Finally (Section 6) we discuss the results we have obtained.

\section{Models for PDF}

As stated above, in this section we briefly introduce two models which describe the scaling evolution of PDF, trying to describe intermittency through the scale variation of characteristic parameters. We consider the model based on a lognormal energy cascade proposed by Castaing et al. 15. 16; the second model describes the PDF through a sequence of stretched exponentials and can be formally derived through the theory of extreme deviations 17 . 


\subsection{The energy cascade model}

Because of the idea of self-similarity underlying the energy cascade process in turbulence, Castaing and co-workers 15 introduced a model which tries to characterize the behaviour of the PDFs through the scaling law of a parameter describing how the shape of the PDF changes in going towards small scales 16 . In its simplest form the model can be introduced by saying that the PDF of the increments $\delta \psi$ (representing here both velocity and magnetic fluctuations) at a given scale $\tau$, is made by a convolution of the typical Gaussian distribution $P_{G}(\delta \psi, \sigma)=(\sqrt{2 \pi} \sigma)^{-1} \exp \left(-\delta \psi^{2} / 2 \sigma^{2}\right)$, with a function $G_{\tau}(\sigma, \lambda)$ which represents the weight of the gaussian distribution characterized by the standard deviation $\sigma$ :

$$
P_{\tau}(\delta \psi, \lambda)=\int G_{\tau}(\sigma, \lambda) P_{G}(\delta \psi, \sigma) d \sigma
$$

where $\lambda$ is a parameter representing the width of $G_{\tau}$.

In the usual approach where the energy cascade is introduced through a fragmentation process, $\sigma$ is directly related to the local energy transfer rate $\epsilon$. In a self-similar situation, where the energy cascade generates only a scaling variation of $\sigma=\left\langle\delta \psi^{2}\right\rangle^{1 / 2}$ according to the classical Kolmogorov's picture $\mathbb{E}$, $G_{\tau}(\sigma)$ reduces to a Dirac function $G_{\tau}(\sigma)=\delta\left(\sigma-\sigma_{0}\right)$. In this case from eq. (11) a Gaussian distribution $P_{\tau}(\delta \psi)=P_{G}\left(\delta \psi, \sigma_{0}\right)$ is retained. On the contrary when the cascade is not strictly self-similar, the width of the distribution $G_{\tau}$ is different from zero. In this way the scaling behaviour of the parameter $\lambda$ (which takes into account the height of the PDFs wings) can be used to characterize intermittency.

\subsection{A model coming from Extreme Deviations Theory}

Recently Frisch and Sornette17, have built up a more general fragmentation model which is based on the extreme deviations theory (EDT), which yield non-gaussian PDF as the number of fragments increases. Let us consider a series of random events $x_{i}$, independent and distributed according to a probability density $p(x)$. According to the central limit theorem, the sum $y=\sum_{i=1}^{n} x_{i}$ is distributed according to a gaussian function, that is fluctuations $y / n$ are $O\left(n^{-1 / 2}\right)$. On the contrary EDT deals with events where fluctuations are $O(1)$, with a finite value $n$ of fragments and large values of $y$. The theory allows us to refgver the asymptotic shape of the PDF $P_{n}(y)$ starting from $p(x)$. The authors 4 consider the general case where $p(x)=\exp [-f(x)]$, and $f(x)$ is differentiable and increases quite rapidly as $x \rightarrow \infty$. Assuming that the 
second derivative is positive $f^{\prime \prime}(x)>0$ and that $x^{2} f^{\prime \prime}(x) \rightarrow \infty$ (for large $x$ ), the asymptotic form for the PDF of $y$ is given by

$$
P_{n}(y) \sim \exp [-n f(x / n)] .
$$

The probability conditioned on $x_{i}$ whose sum is $y$, is localized for large $y$ in a "democratic" manner near the value for which $y / n \simeq x_{i} \simeq x_{2} \simeq \ldots \simeq x_{n}$. If during the fragmentation process the fragments have probability $p(x) \sim$ $\exp \left(-C x^{\gamma}\right)$ with $\gamma>0$, and $y=\log S$, where $S=w_{1} w_{2} \ldots w_{n}$ (that is $x_{i}=$ $\left.\log w_{i}\right)$, then

$$
P(S) \sim \exp \left(-C n S^{\gamma / n}\right) .
$$

From the turbulence point of view, if at the scale $\tau$ the value of each $\delta \psi$ is due to the product of fragments derived from structures at larger scale, from EDT we must expect a PDF as a stretched exponential

$$
P(\delta \psi) \sim \exp \left[-\beta(\tau)|\delta \psi|^{\mu(\tau)}\right]
$$

where $\beta(\tau)$ and $\mu(\tau)$ are the parameter we must fix from data.

With these models in mind we will discuss now what kind of results are obtained for plasma turbulence.

\section{A shell model for MHD turbulence}

Turbulence is a phenomenon in which chaotic dynamics and power law statistics coexist. In general, due to the high Reynolds numbers involved in realistic situations, direct numerical simulations of 3D turbulence is not possible. In order to avoid this problem and in order to capture the gross features of turbulence, an alternative approach can be followed by investigating reduced models of turbulence, such as shell models 18 . In these models the wave vector domain is spaced exponentially through $k_{n}=k_{0} 2^{n}(n=1, \ldots, N)$ and the nonlinear dynamics is reproduced by using a single complex variable for each $n$-th shell, that is $u_{n}(t)$ for the velocity field and $b_{n}(t)$ for the magnetic field. The quadratic nonlinear interactions between modes are then recovered by imposing that: i) the dynamical equations for each shell variable must contain the same quadratic terms of the original equations; ii) the quadratic invariants of equations must be correctly preserved in the inviscid case; iii) the nonlinear coupling occur between nearest or next nearest shells, modeling local interactions in the $k$ space. By using these criteria an MHD shell model which is the analogous of the GOY model for hydrodynamics is easily obtained (see-22 and 19 for a complete review on shell models): 


$$
\begin{aligned}
\frac{d u_{n}}{d t} & =i k_{n}\left[\left(u_{n+1} u_{n+2}-b_{n+1} b_{n+2}\right)-\frac{1}{4}\left(u_{n-1} u_{n+1}-b_{n-1} b_{n+1}\right)+\right. \\
& \left.-\frac{1}{8}\left(u_{n-2} u_{n-1}-b_{n-2} b_{n-1}\right)\right]^{*}-\nu k_{n}^{2} u_{n}+f_{n} \\
\frac{d b_{n}}{d t} & =i k_{n}\left[\frac{1}{6}\left(u_{n+1} b_{n+2}-b_{n+1} u_{n+2}\right)+\frac{1}{6}\left(u_{n-1} b_{n+1}-b_{n-1} u_{n+1}\right)+\right. \\
& \left.+\frac{1}{6}\left(u_{n-2} b_{n-1}-b_{n-2} u_{n-1}\right)\right]^{*}-\eta k_{n}^{2} b_{n}+g_{n}
\end{aligned}
$$

where $\nu$ and $\eta$ are respectively the kinematic viscosity and the resistivity, and $f_{n}$ and $g_{n}$ are forcing terms. Equations (何) and (5), in the inviscid and unforced case, conserve the total energy

$$
E(t)=\frac{1}{2} \sum_{n=1}^{N}\left(\left|u_{n}\right|^{2}+\left|b_{n}\right|^{2}\right)
$$

the cross-helicity

$$
H_{c}(t)=\frac{1}{4} \sum_{n=1}^{N} \operatorname{Re}\left(u_{n} \cdot b_{n}^{*}\right)
$$

and the magnetic helicity

$$
H(t)=\sum_{n=1}^{N}(-1)^{n} \frac{\left|b_{n}\right|^{2}}{k_{n}}
$$

Apart for other features, shell models share with original equations scaling laws of the structure functions $S_{p}(n)=\left\langle\left|x_{n}\right|^{p}\right\rangle \sim k_{n}^{-\zeta_{p}}$ where $x_{n}$ is either $u_{n}$ and $b_{n}$. Scaling exponents $\zeta_{p}$ deviates from the linear Kolmogorov scaling as a consequence of the intermittency in the dynamical system.

We solved numerically the system of equations for the shell model, by using the following set of parameters: $N=23$ shells, $\nu=\eta=10^{-9}$, the integration step $d t=5 \times 10^{-5}$. The forcing term acts only on the velocity variables (i.e. $\left.g_{n}=0\right)$ at large scales $(n=1,2)$, the magnetic field being generated by a kind of dynamo effect which is at work in the MHD shell model 12. The forcing, which is assumed to be random, is calculated according to a Langevin equation 
$d f_{n} / d t=-f_{n} \tau_{0}+\theta$, where $\tau_{0}$ is the characteristic time of the largest scales, and $\theta$ is a gaussian white-noise. PDFs for both the velocity and magnetic variables have been obtained and are shown in figures 1 and 3 for different shells. As it can be seen PDFs depends on the scale $\ell_{n} \sim k_{n}^{-1}$. They are almost Gaussian at the large scales (small shells $k_{n}$ ) and are increasingly non-Gaussian at small scales (large shells $k_{n}$ ).

In order to make a quantitative analysis of the energy cascade leading to the process just described, we have tried to fit the distributions by using, for the model (11), the log-normal ansatz 15

$$
G_{\tau}(\sigma) d \sigma=\frac{1}{\lambda(\tau) \sqrt{2 \pi}} \exp \left[-\frac{\ln ^{2}\left(\sigma / \sigma_{0}\right)}{2 \lambda^{2}(\tau)}\right] d(\ln \sigma)
$$

even if also other functions gives rise to results not really different. The parameter $\sigma_{0}$ represents the most probable value of $\sigma$, while $\lambda^{2}(\tau)=\left\langle(\Delta \ln \sigma)^{2}\right\rangle$ is the width of the log-normal distribution of $\sigma$.

We have fitted the expression (11) on the PDFs obtained in the shell model for both velocity and magnetic intensity, and we have obtained the corresponding values of the parameter $\lambda^{2}$. The values of the parameters $\sigma_{0}$, which do not display almost any variation with $\ell_{n}$ are reported in table 1 . In figure 1 we plot, as full lines, the curves relative to the fit. As can be seen the scaling behaviour of PDFs in all cases is very well described by the function (11). From the fit, at each scale $\ell_{n}$, we get a value for the parameter $\lambda^{2}\left(\ell_{n}\right)$, and in figure 2 we report the scaling behaviour of $\lambda^{2}\left(\ell_{n}\right)$.

The scaling evolution of PDFs have been analysed also using the stretched exponential model $P\left(\delta x_{\ell}\right) \sim \exp \left[-\beta\left(\delta x_{\ell}\right)^{\mu}\right]$; the results of the fit are displayed in figure 3 as a full line. The parameters $\mu$ found from the fit are shown in figure 1 as a function of the scale $\ell_{n}$, for both $x_{\ell_{n}}=u_{n}$ and $x_{\ell_{n}}=b_{n}$. As can be seen, a power law $\mu\left(\ell_{n}\right) \sim \ell_{n}^{a}$ is found in a very wide range for the magnetic field; the range is less wide in the case of the velocity field, for wich a saturation of $\mu$ is reached. The minima values for $\mu$ are reported in the table 2, together with the exponents $a$ found for the power laws.

As we will show in the next two Sections, both models are able to capture intermittency also for turbulence in non homogeneous situations, namely the Solar Wind and a fusion plasma. To avoid superpositions we describe for the Solar Wind only the results obtained with log-normal ansatz, while for the fusion plasma we describe only the results obtained with the stretched exponentials, even if in both 1 and 2 the reader can find the characteristic parameters obtained through the fit of both models for both samples. 


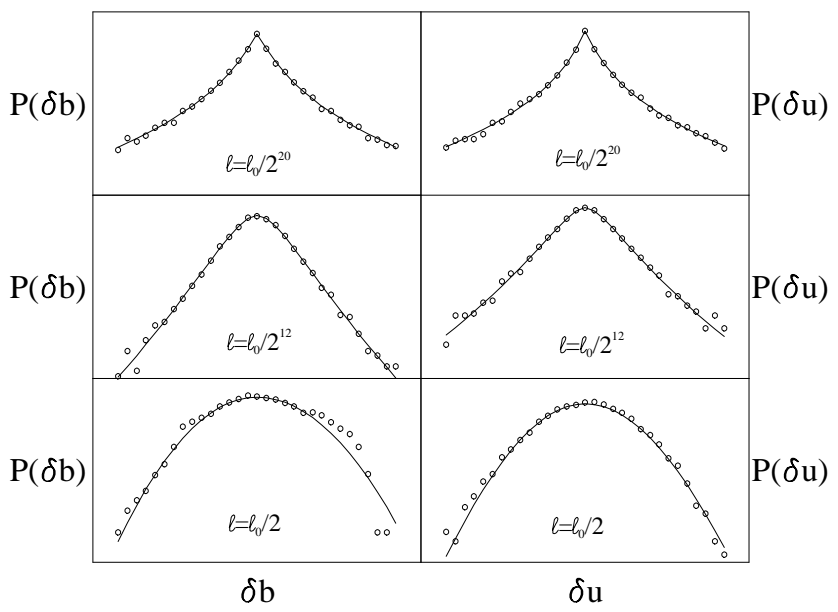

Figure 1: The PDFs of the coefficients $b_{n}$ and $u_{n}$ for three different values of $n$. The fit with the log-normal model by Castaing et al. (1) is superimposed as a full line.

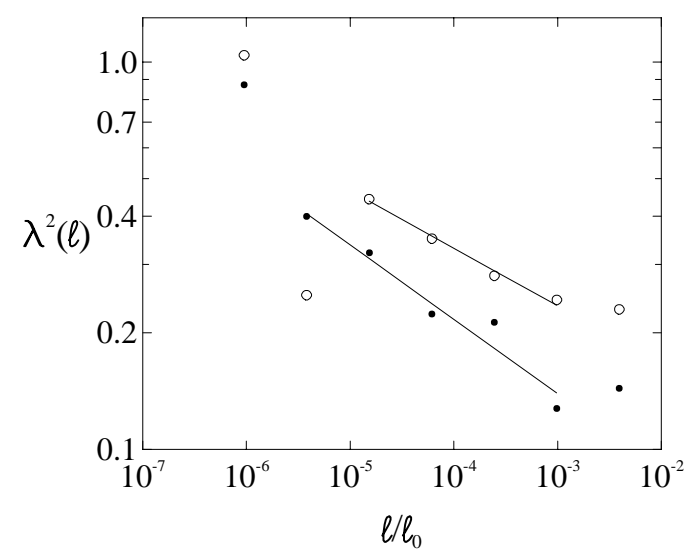

Figure 2: The values of the parameter $\lambda^{2}$ as a function of the scale $\ell / \ell_{0}$. Black circles are for the magnetic field, while the white circles are for the velocity. 


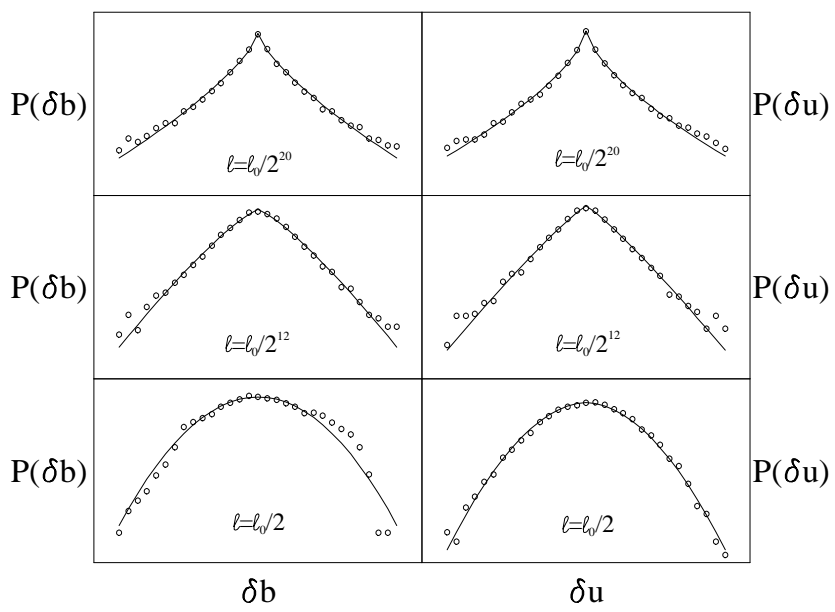

Figure 3: The PDFs of the coefficients $b_{n}$ and $u_{n}$ for three different values of $n$. The fit with the stretched exponentials functions is superimposed as a full line.

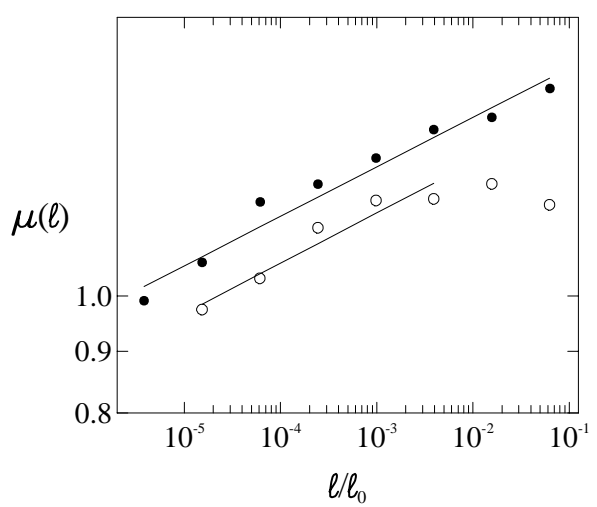

Figure 4: The values of the exponent $\mu$ as a function of the scale $\ell / \ell_{0}$. Black circles are for the magnetic field, while the white circles are for the velocity. 


\section{Solar Wind Observations}

The satellite observations of both velocity and magnetic field in the interplanetary space, offer us an almost unique possibility to gain information on the turbulent MHD state in a very large scale range, say from $1 \mathrm{AU}$ (Astronomical Unit) down to $10^{3} \mathrm{~km}$. Here we limit to analyse only plasma measurements of the bulk velocity $V(t)$ and magnetic field intensity $B(t)$. We based our analysis on plasma measurements as recorded by the instruments on board Helios 2 during its primary mission in the inner heliosphere. The analysis period refers to the first four months of 1976 when the spacecraft orbited from $1 \mathrm{AU}$, on day 17 , to $0.29 \mathrm{AU}$ on day 108 . The original data were collected in $81 \mathrm{~s}$ bins and we choose a set of subintervals of two days each. The subintervals were selected separately within low speed regions and high speed regions selected in a standard way according to a threshold velocity 4 . For each subinterval we calculated the velocity and magnetic increments at a given scale $\tau$ through $\delta V_{\tau}=V(t+\tau)-V(t)$ and $\delta B_{\tau}=B(t+\tau)-B(t)$, which represent characteristic fluctuations across eddies at the scale $\tau$. Then we normalize each variable to the standard deviation within each subinterval $\delta v_{\tau}=\delta V_{\tau} /\left\langle\delta V_{\tau}^{2}\right\rangle^{1 / 2}$ and $\delta b_{\tau}=\delta B_{\tau} /\left\langle\delta B_{\tau}^{2}\right\rangle^{1 / 2}$ (where brackets represent average within each subinterval at the scale $\tau$ ). We calculate the PDFs at 11 different scales logarithmically spaced $\tau=\Delta t 2^{n}$, where $n=0,1, \ldots, 10$ and $\Delta t=81 \mathrm{~s}$. We collect the number of events within each bins by using 31 bins equally spaced in the range within 3 times the standard deviation of the total sample. Before we mixed the different subperiods belonging to a given class (high or low speed streams), we tested for the fact that the gross features of PDFs shape does not change in different subintervals. Then our results for high and low speed streams are representative of what happens at the PDFs.

The results are shown in figures 5 and 6 , where we report the PDFs of both velocity and magnetic intensity for both the high speed streams and the slow speed streams. At large scales the PDFs are almost Gaussian, and the wings of the distributions grow up as the scale becomes smaller. This is true in all cases, say for both types of wind. Stronger events at small scales have a probability of occurrence greater than that they would have if they were distributed according to a gaussian function. This behaviour is common for intermittency as currently observed in fluid flows 0 and in the solar wind turbulence 2014 .

A quantitative analysis of the energy cascade has been made by using the log-normal ansatz15 (5). We have fitted the expression (1) on the experimental PDFs for both velocity and magnetic intensity, and we have obtained the corresponding values of the parameter $\lambda^{2}$. The values of the parameters $\sigma_{0}$, 


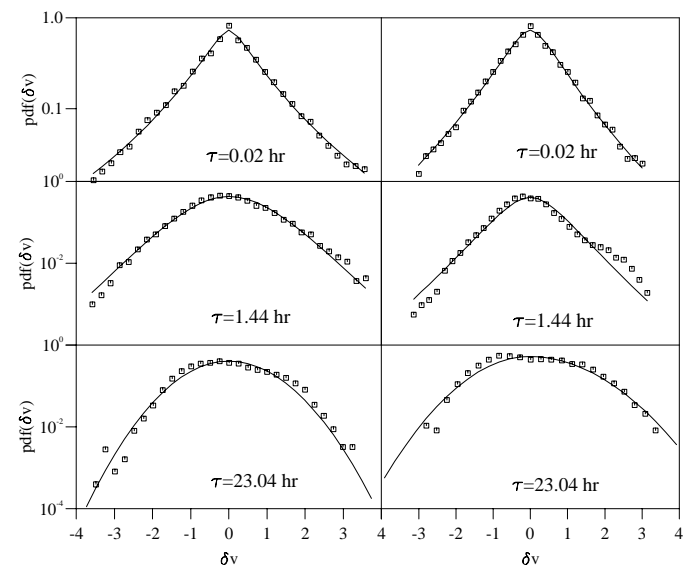

Figure 5: The scaling behaviour of the PDF for $\delta v_{\tau}$ as calculated from the experimental data (white symbols) in the fast (left) and slow (right) streams. The full lines represent the fit obtained through the model by Castaing et al. (1), as described in the text.

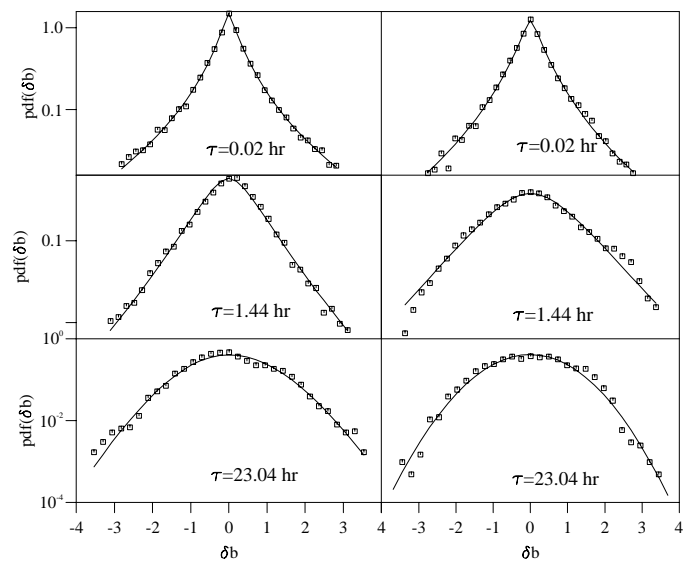

Figure 6: The scaling behaviour of the PDF for $\delta b_{\tau}$ as calculated from the experimental data (white symbols) in the fast streams. Again, the full lines represent the fit obtained through the model by Castaing et al. (1), as described in the text. 


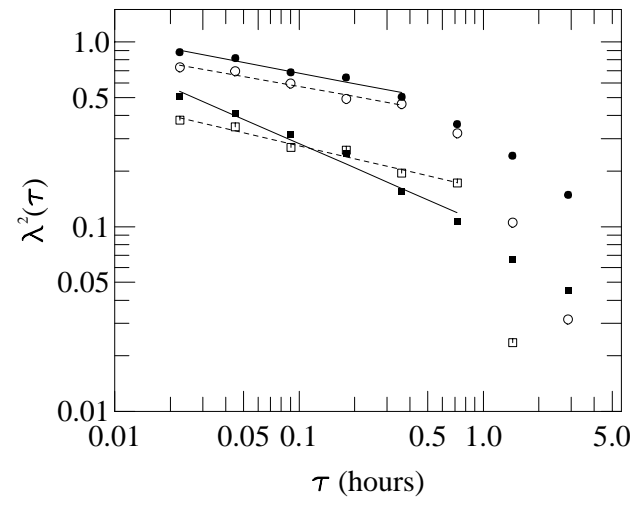

Figure 7: We show the scaling behavior of $\lambda^{2}(\tau)$ vs. $\tau$ for both fast (black symbols) and slow (open symbols) streams. Circles refer to the magnetic field intensity, squares refer to the bulk velocity.

which again do not display almost any variation with $\tau$ are reported in table 1. In figures 5 and 6 we plot, as full lines, the curves relative to the fit. As can be seen the scaling behaviour of PDFs in all cases is very well described by the function (1). From the fit, at each scale $\tau$, we get a value for the parameter $\lambda^{2}(\tau)$, and in figure 7 we report the scaling behaviour of $\lambda^{2}(\tau)$ for both high and low speed streams. Starting from a very low value at the scales of about 1 day, $\lambda^{2}$ increases abruptly to $\lambda^{2} \simeq 10^{-1}$ at about 2 hours, and finally a scaling law starts to become evident up to $\Delta t=81 \mathrm{sec}$. In this last range, which corresponds roughly to what is usually called the "Alfvénic range", we fitted the parameter with a power law $\lambda^{2}(\tau) \sim \tau^{-\alpha}$. The values of $\alpha$ obtained in the fitting procedure and the corresponding range of scales, are reported in the table 1. In the same table, we report the values of the maximum value of $\lambda^{2}(\tau)$ in the range of fit, namely $\lambda_{\max }^{2}$, which is an indication for the strenght of the intemittency 10.21 .

Looking at figure 7 and at $\lambda_{\max }^{2}$, it can be seen that both in fast and in slow streams magnetic field intensity is more intermittent than bulk velocity (values of $\lambda^{2}$ are at least two times larger for magnetic field intensity than for velocity). The same indications comes from 2D MHD direct simulations 9.12, and in different analysis of solar wind intermittency 22. The values of $\lambda^{2}(\tau)$ are more or less the same for magnetic field intensity both in fast and in slow wind. This is due to the fact that magnetic field intensity fluctuations 
are related to compressive fluctuations, which should have the same nature in both types of wind. The bulk velocity fluctuations on the contrary are more intermittent at small scales $(81 \mathrm{sec})$ in the fast wind and at large scale $(\simeq 1$ hour) in the slow wind. From table 1 it appears that the value of $\alpha$ is not universal, a result which has also been found in fluid flows 15 . The fact that the value of $\alpha$ for the velocity fluctuations in slow wind is the same as the value of $\alpha$ for magnetic field intensity suggests that intermittent structures in slow wind are perhaps mainly associated with compressive fluctuations. On the contrary the different value of $\alpha$ found in fast wind evidences a different nature of velocity fluctuations in fast wind, perhaps related to the fact that such fluctuations are mainly incompressible. This result is in agreement with what has been found by Veltri and Mangeney 22. These authors found that in fast wind the more intermittent structures are tangential discontinuities with almost no variation in magnetic field intensity, while in slow wind the most intermittent structures are shock waves, which display the same behaviour in bulk velocity and magnetic field intensity.

\section{The Reverse Field Pinch: RFX experiment}

In this section we report evidences for the presence of intermittency in another type of magnetized fluid, namely a plasma of interest for controlled thermonuclear fusion research, confined in reversed field pinch (RFP) configuration.

The RFP is a toroidal configuration of magnetic fields which is proposed as an alternative to the tokamak for confining fusion grade plasmas 23 . The configuration is characterized by toroidal and poloidal magnetic field components of comparable magnitude (in a tokamak the field is mainly toroidal). The configuration is a near-minimum energy state to which a plasma relaxes under proper constraints 24. The toroidal field changes sign in the outer part of the plasma, a feature which gives the name to the configuration. Such field reversal, which improves the MHD stability of the configuration, is spontaneously generated by the plasma, and is maintained against resistive diffusion by the dynamo process 2 . This is achieved through the action and nonlinear coupling of several resistive magnetohydrodynamic modes, which give rise to a high level of magnetic turbulence (of the order of $1 \%$ of the average field in present day experiments, i.e. two orders of magnitude larger than in tokamaks). This high fluctuation level makes the RFP very suited for the study of MHD turbulence properties, mainly for their magnetic part. The magnetic turbulence has been demonstrated to be the main cause of energy and particle transport in the RFP core, whereas at the edge its contribution is still under investigation. In this region the electrostatic turbulence has been proved to 


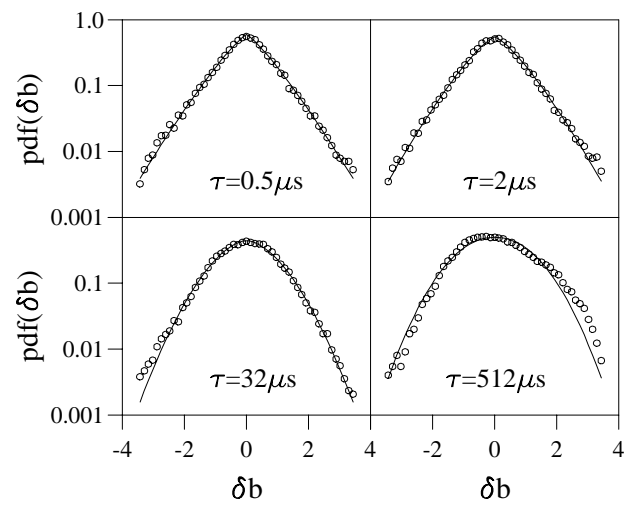

Figure 8: The scaling behaviour of the PDF of the magnetic fluctuation $\delta b_{\tau}$ measured in the RFX experiment. The full lines represent the fit obtained through the stretched exponential model, as described in the text.

give an important contribution to the particle transport 25 .

The measurements have been obtained in the RFX experiment, which is the largest RFP presently in operation (major radius $2 \mathrm{~m}$, minor radius 0.457 m) 26. RFX is designed to reach a plasma current of 2 MA, and currents up to $1 \mathrm{MA}$ have been obtained up to now. The measurements were performed in low currents discharges $(300 \mathrm{kA})$ using a magnetic probe inserted in the edge plasma. The probe consists of a coil housed in a boron nitride measuring head. The coil measures the time derivative $\partial_{t} B$ of the radial component $B(t)$ of the magnetic field. The radial direction in this case goes from the core plasma to the edge. The sampling frequency of the measurements is $2 \mathrm{MHz}$. Measurements have been collected at different values of the probe insertion $X=r / a$ into the plasma, $X=0$ being the position of the inner convolution of the RFX graphite first wall, $r$ being the probe radial position and $a$ being the radius of the ring. Different measurements are also identified through a shot number. In RFX two different components of the magnetic fluctuations can be identified: a localised and stationary magnetic perturbation, originated by the tearing modes responsible for the dynamo which tend to be phase-locked and locked to the wall 27 , and an high frequency broadband activity, which is investigated here. All measurements presented were made away from the stationary perturbation. 


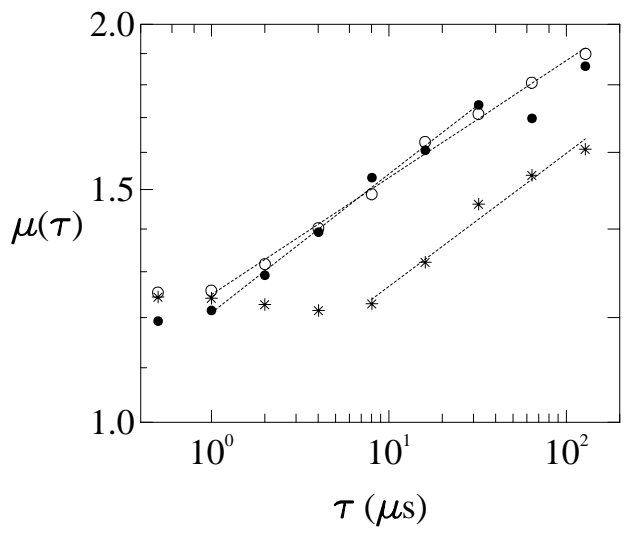

Figure 9: We show the scaling of $c(\tau)$ vs. $\tau$ for the RFX magnetic field at different insertion values: $X=0.97$ (black circles), $X=0.95$ (white circles) and $X=0.91$ (stars).

We calculated the characteristic magnetic fluctuations at the scale $\tau$, namely $\delta B(\tau)=B(t+\tau)-B(t)$. For each position within the device, we can study the statistical behaviour of fluctuations at different scales $\tau$. In figure 8 we report the PDFs of $\delta b_{\tau}=\delta B_{\tau} /\left\langle\delta B_{\tau}^{2}\right\rangle^{1 / 2}$ at different scales for a given value of $X$. As it can be seen the PDFs do not collapse to a single curve, but follow the usual characteristic scaling behaviour evidenced also in solar wind measurements. This behaviour is visible for all the values of the insertion $X$. A fit to the PDFs we obtained has been made by using a stretched exponential, namely $P\left(\delta b_{\tau}\right) \sim \exp \left[-\beta\left(\delta b_{\tau}\right)^{\mu}\right]$, and their scaling behaviour is then investigated by looking at the scaling laws of both the parameters $\beta(\tau, X)$ and $\mu(\tau, X)$. The parameter $\mu$ follows a power law in a certain range of scales, that is: $\mu(\tau, X) \sim \tau^{a(X)}$, even near the external wall (figure 5 ).

The scaling exponent $a(X)$ (see table 2) remains near to the value 0.1 in the different shots, and the range of scales where a scaling for the parameter $\mu(\tau, X)$ is visible is larger for measurements near the first wall with respect to measurements inside the device. The "strenght" of the intermittency effects can be compared for the different insertion values $X$, by comparing the minimum values found for the exponent $\mu(\tau)$ in each shot. As can be seen from the table (2), such values increase going toward the core, showing that the intermittency is stronger near the wall 21 . 
Table 1: We report the values of the parameters $\sigma_{0}, \lambda_{\max }^{2}$ and $\alpha$ obtained in the fitting procedure for $\lambda^{2}(\tau)$ of the log-normal model in the shell model data, solar wind and RFX (shot 8414 ). We also report the range of scales where the fit has been done. For the shell model, the range of fit is in $\ell / \ell_{0}$ units.

\begin{tabular}{ccccc} 
& $\sigma_{0}$ & $\lambda_{\max }^{2}$ & $\alpha$ & Scales (SW: hours) \\
\hline$u_{S M}$ & $0.83 \pm 0.04$ & $0.44 \pm 0.10$ & $0.15 \pm 0.07$ & $1.5 \times 10^{-5} \div 10^{-3}$ \\
$b_{S M}$ & $0.79 \pm 0.04$ & $0.40 \pm 0.10$ & $0.19 \pm 0.06$ & $3.8 \times 10^{-6} \div 10^{-3}$ \\
$B_{S W}$ (fast) & $0.85 \pm 0.05$ & $0.88 \pm 0.04$ & $0.19 \pm 0.02$ & $0.02 \div 0.72$ \\
$B_{S W}$ (slow) & $0.90 \pm 0.05$ & $0.73 \pm 0.04$ & $0.18 \pm 0.03$ & $0.02 \div 0.72$ \\
$V_{S W}$ (fast) & $0.90 \pm 0.05$ & $0.51 \pm 0.04$ & $0.44 \pm 0.05$ & $0.02 \div 1.44$ \\
$V_{S W}$ (slow) & $0.95 \pm 0.05$ & $0.37 \pm 0.03$ & $0.20 \pm 0.04$ & $0.02 \div 1.44$ \\
$R F X_{8414}$ & $1.01 \pm 0.02$ & $0.21 \pm 0.01$ & $0.42 \pm 0.03$ & $1 \mu s \div 36 \mu s$
\end{tabular}

Table 2: The values of $\mu_{\min }$ and the scaling exponents $a$, obtained for the scaling of the exponents $\mu$ of the stretched exponential fit of the data, from shell model, the RFX and the solar wind. The scale ranges in which a power law has been found are also shown. In the RFX, different shots atdifferent insertions $X=r / a$ are displayed. Two inner shots $(8425$ and 8426) do not display clear scaling laws. However, we report the minima of $\mu$. For the shell model, the range of fit is in $\ell / \ell_{0}$ units.

$\begin{array}{llll}X & a & \text { Scales }(\mathrm{RFX}: \mu s)\end{array}$

\begin{tabular}{ccccc}
\hline$u_{S M}$ & - & $0.97 \pm 0.03$ & $0.04 \pm 0.01$ & $1.5 \times 10^{-5} \div 3.9 \times 10^{-3}$ \\
$b_{S M}$ & - & $0.99 \pm 0.03$ & $0.04 \pm 0.01$ & $3.8 \times 10^{-6} \div 6.3 \times 10^{-2}$ \\
$R F X_{8414}$ & 0.97 & $1.19 \pm 0.02$ & $0.10 \pm 0.01$ & $1 \div 36$ \\
$R F X_{8417}$ & 0.96 & $1.25 \pm 0.01$ & $0.09 \pm 0.01$ & $1 \div 128$ \\
$R F X_{8420}$ & 0.91 & $1.23 \pm 0.01$ & $0.10 \pm 0.01$ & $8 \div 128$ \\
$R F X_{8425}$ & 0.90 & $1.50 \pm 0.02$ & - & - \\
$R F X_{8426}$ & 0.86 & $1.55 \pm 0.03$ & - & - \\
$B_{S W}($ fast $)$ & - & $0.74 \pm 0.01$ & $0.16 \pm 0.01$ & $0.02 h \div 24 h$ \\
$B_{S W}$ (slow) & - & $0.81 \pm 0.02$ & $0.16 \pm 0.02$ & $0.02 h \div 24 h$ \\
$V_{S W}$ (fast) & - & $0.92 \pm 0.02$ & $0.12 \pm 0.02$ & $0.02 h \div 24 h$ \\
$V_{S W}$ (slow) & - & $1.04 \pm 0.03$ & $0.11 \pm 0.01$ & $0.02 h \div 24 h$
\end{tabular}




\section{Discussion and conclusions}

The departure from gaussianity of the PDFs of fields increments is well known in fluid turbulence, and has been pointed out in MHD plasmas. The data presented in this paper clearly confirm this peculiar behavior, showing that intermittency strongly affects the scaling behavior of MHD turbulence. The analysis of data with the two models presented here has a duplex interest: i) it is an experimental test for the theoretical models; ii) it gives a quantitative characterization of intermittency in different physical systems, leading to the the possibility of a comparison between them.

For the first point, we showed and discussed in detail here the results obtained when we used the log-normal model to describe intermittency in the solar wind turbulence, and the stretched exponential model to describe intermittency in RFX plasma. However it can be shown that both models are able to capture the scaling behavior of PDFs in both the solar wind and RFX experiments, just as in the case of the MHD shell model. Very good fits are obtained, and very close values of the $\chi^{2}$ are found in both cases 28 . In table

1 we report the results relative to the fit of PDFs obtained in RFX plasma (namely the shot number 8414) with the model by Castaing et al. 15 . Other samples give similar results. In table 2 we report the results obtained when we fit the PDFs calculated with the dataset of the solar wind turbulence, with the stretched exponential model.

Concerning the second point, we first observe that the two models give consistent results. The similar role played by $\lambda_{\max }^{2}$ in the log-normal model and $\mu_{\min }$ in the stretched exponential model, as well as the slopes of the power-low scaling of $\lambda^{2}$ and $\mu$, is evident. We can thus look at such parameters to compare the intermittency of the magnetic field in both experimental datasets we analysed. As a first comparison we can observe, looking directely at the PDFs shape of magnetic fluctuations, that intermittency in solar wind is stronger than in the RFX experiment plasma. The values of both parameters $\lambda_{\max }^{2}$ and $\mu_{\text {min }}$ (see tables 1 and 2) confirm such result. Clear scaling laws are found for the parameters of both models, so that it seems difficult to prefer one model with respect to the other on the basis of our analysis.

Even if the experimental results we presented do not give indications wheter one model is better than the other in fitting experimental results, the stretched exponential is a more flexible function with respect to (11). This is only a "technical" consideration and does not involve a theoretical preference. From our experience the code developed for the fit with the stretched exponential is more performant then the code developed by using the lognormal model. It is however worthwhile to compare intermittency found in 
real datasets with time intermittency found in the simplified models for MHD turbulence. Looking at the PDFs in figures 1, 3, 5 and 6, we can realise that intermittency in the shell model is similar to that obtained in the solar wind, that is turbulence in the shell model is more intermittent than turbulence in RFX plasma. Furthermore looking at the values of $\mu_{\min }$, it can be seen that magnetic turbulence in the solar wind is more intermittent than in the shell model, while for the velocity the intermittency is comparable in both cases.

To conclude we would like to remark that numerical simulations of MHD

equations in $2 \mathrm{D}$ configurations 10 display strong intermittency which has been successfully described by the model introduced by Castaing et al. 15 .

\section{Acknowledgements}

We are grateful to H. Rosenbauer and R. Schwenn for making the Helios plasma data available to us.

\section{References}

1. Frisch, U., Turbulence: the legacy of A. N. Kolmogorov, Cambridge University Press, 1995.

2. Biskamp, D., Nonlinear Magnetohydrodynamics, Cambridge University Press, 1997.

3. Burlaga, L.F., J. Geophys. Res., 96, 5847, 1991.

4. Marsch, E., and Liu, S., Ann. Geophys., 11, 227, 1993.

5. Carbone, V., Veltri, P., Bruno R., Phys. Rev. Lett., 75, 3110, 1995.

6. Carbone, V., Bruno R., Veltri, P., Geophys. Res. Lett., 23, 121, 1996.

7. Ruzmaikin, A.A., Feynman, J., Goldstein, B.E., and Smith E.J., J. Geophys. Res., 100, 3395, 1995.

8. Horbury, T.A., Balogh, A., Forsyth, R.J., and Smith, E.J., Adv. Space Phys., 19, 847, 1997.

9. Politano, H., Pouquet, A., and Carbone, V., Europhys. Lett., 43, 516, 1998.

10. Sorriso-Valvo, L., Carbone, V., Veltri, P., Politano, H. and Pouquet, A., Europhys. Lett., 51, 520, (2000).

11. Carbone, V., Europhys. Lett., 27, 581, 1994.

12. Giuliani, P. and Carbone, V. Europh. Lett., 43, 527 (1998)

13. Marsch, E., and Tu, C.Y., Ann. Geophys., 12, 1127, 1994.

14. Sorriso-Valvo L., Carbone, V., Consolini, G., Bruno, R. and Veltri, P., Geophys. Res. Lett., 26, 1801 (1999).

15. Castaning, B., Gagne, Y., and Hopfinger E.J., Physica D, 46, 177, 1990.

16. Vassilicos, J.C., Nature, 374, 408, 1995. 
17. Frisch, U., and Sornette, D., J. Phys. I France, 7, 1155 (1997).

18. Bohr, T., Jensen, M. H., Paladin G., and Vulpiani, A., Dynamical Systems approach to Turbulence, Cambridge University Press, 1998.

19. Giuliani, P., in Non-linear MHD Waves and Turbulence, T. Passot and P. L. Sulem Eds., Lecture Notes in Physics, Springer-Verlag, 1999.

20. Marsch, E., and Tu, C.Y., Nonlin. Proc. in Geophys., 4, 101, 1997.

21. Carbone, V., Sorriso-Valvo, L., Veltri, P., Martines, E. and Antoni, V., Phys. Rev. E, 62, R49 (2000).

22. Veltri, P., and Mangeney, A., Scaling laws and intermittent structures in solar wind MHD turbulence, Proceedings of Solar Wind 9, 1999.

23. Bodin, H. A. B., Nucl. Fusion, 30, 1717 (1990).

24. Taylor, J. B., Phys. Rev. Lett., 33, 1139 (1974).

25. Antoni, V. ,Cavazzana, R., Desideri, D. ,Martines, E., Serianni, G. and Tramontin, L., Phys. Rev. Lett. 80, 4185 (1998).

26. Fellin, L., Kusstatscher, P. and Rostagni, G., Fusion Eng. Des., 25, 315 (1995).

27. Antoni, V. et al., Proceedings of the 22nd EPS Conference on Controlled Fusion and Plasma Physics, Bournemouth (1995), part IV, p.181.

28. Sorriso-Valvo, L., Tesi di Laurea, unpublished (1998). 